\title{
Experimental Verification of the Magnetic Field Topography inside a small Hall Thruster
}

\author{
Maciej Jakubczak ${ }^{1,2}$, Jacek Kurzyna ${ }^{1}$, Arsenii Riazantsev ${ }^{1}$ \\ ${ }^{1}$ Institute of Plasma Physics and Laser Microfusion, Hery 23, 01-497,Warsaw, Poland, maciej.jakubczak@ifpilm.pl \\ ${ }^{2}$ Faculty of Physics, Warsaw University of Technology, Koszykowa 75, 00-662, Warsaw, Poland
}

\begin{abstract}
The magnetic circuit of a $500 \mathrm{~W}$ class Hall thruster, an electric propulsive device for spacecraft, was characterized experimentally and the results compared with simulation in order to verify the design. The commercial 3D gaussmeter, which was used in this work, was additionally recalibrated to compensate for translation and rotation of individual Hall sensors inside the probe. The Stokes stream function approach was applied to reconstruct the magnetic field topography in the thruster. The procedure, carried out on four different cases, yielded very good agreement between simulations and measurements, even for cusped configurations. Presented technique could be used as a robust method of verification of new magnetic circuit designs not only for Hall thrusters but also for a wide class of plasma devices for which detailed knowledge about actual distribution of magnetic field is crucial for optimization.
\end{abstract}

Keywords: Hall thruster, magnetic field, topography, verification.

\section{INTRODUCTION}

The notion of electric propulsion (EP) is relevant to a wide class of jet-like engines in which acceleration of expelled mass is provided by direct or indirect application of electric power. In this way the source of energy needed for speedingup a propellant is decoupled from it and usually, due to the use of photovoltaic panels, can be considered as external with respect to the spacecraft. In comparison to classic chemical rocket engines, utilization of electric power typically brings significant reduction of the propellant mass needed to gain the same spacecraft velocity $\Delta V$, owing to overcoming thermodynamic limitation for maximum speed of the expelled particles. The speed of ionized particles accelerated by electric thruster can be several times (5-10) greater than that of gas expelled by classic rocket engines. Not mentioning other advantages, the outlined two basic features - mass reduction and utilization of external energy - make electric propulsion so attractive for space thruster technology.

Electric propulsion covers a wide range of distinct technologies like Hall Effect Thrusters (HET), Ion Gridded Thrusters (IGT), Pulsed Plasma Thrusters (PPT), FieldEmission Electric Propulsion (FEEP), to name only a few, which span a power range from $100 \mathrm{MW}$ to $10 \mathrm{~W}$ [1]. Indeed, recent trend towards miniaturized satellites (e.g., of the CubeSat type) stimulates research of even smaller EP devices with powers of the order of $1 \mathrm{~W}$, suitable for these smallest satellites with very limited power on board [2], [3]. Equipping these satellites with thrusters would enable them to carry out long-duration missions and even work in constellations, e.g. for astrophysical measurements [4].

Hall thruster is one of the first and longest studied electric propulsion devices, with flight heritage dating back to December 1971 (Russian satellite METEOR-18). Since that time numerous models have been in operation providing orbit control capabilities to telecommunication and government satellites, and even serving as a main propulsion engine for orbit raising (e.g., ESA lunar mission SMART-1 in 2003) [5]. Despite extensive research on physics and performance of Hall thrusters that has been already made [6], [7], many groups from both industry and academia pursue new designs and variants of this well-established technology to further improve its efficiency or lifetime.

Operation principle of a Hall thruster, schematically shown in Fig.1., is similar to other closed-drift plasma devices in which electric and magnetic fields induce $\mathbf{E} \times \mathbf{B}$ drift of the charged particles. However, the magnetic field is such that only the electrons are magnetized (i.e., their Larmor radius is much smaller than the width of the discharge channel, of the order of $0.1 \mathrm{~mm}$ ), while its influence on trajectories of much heavier ions is negligible (Larmor radius of the order of several meters). Consequently, the axial mobility of the electrons is reduced and electron density increased in the area of strong magnetic field which facilitates effective ionization of the gaseous propellant (typically $\mathrm{Xe}$ or $\mathrm{Kr}$ ) injected into the discharge channel. The created ions are then accelerated by the electric field and ejected out of the device, producing thrust. New electrons for ionization and neutralization of the beam are constantly supplied by a hollow cathode. 


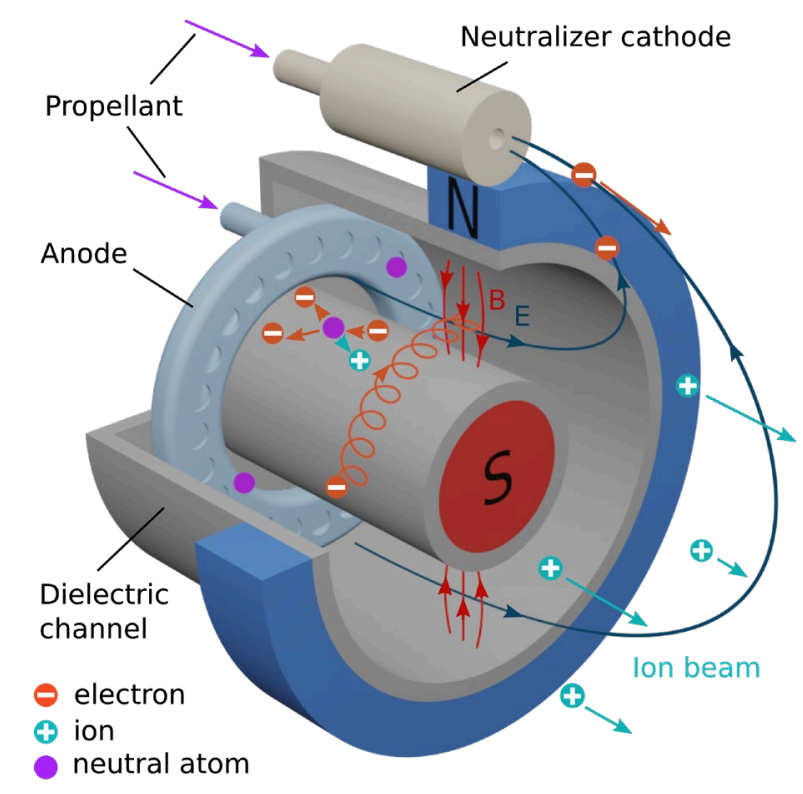

Fig.1. Operation principle of a Hall thruster. Electrons drifting in crossed $\mathbf{E}$ and $\mathbf{B}$ fields facilitate ionization of the gaseous propellant supplied from the bottom of the channel.

The distribution of the electric potential inside the channel determines how well the ions are extracted from the discharge channel, i.e. how many of them leave the thruster without hitting the insulating wall of the channel (important from the point of view of both lifetime and efficiency of the device). Unfortunately, it can be controlled only indirectly by shaping the magnetic field, which controls diffusion of the electrons towards the anode and thus effective plasma resistivity, which in turn affects final distribution of the electric potential inside the plasma. Consequently, recent efforts in the development of Hall thruster technology have been geared largely towards optimization of the magnetic field topography [8].

In the end, any attempt of optimization must be experimentally checked to see whether the specific configuration of the magnetic field (usually modelled numerically) entails an improvement of performance of the thruster. However, it has been noticed that in literature there are very few reported attempts of experimental verification of the produced magnetic field itself. While numerical simulations of the magnetic field are quite precise, the end product depends on the magnetic properties of the elements of the thruster, which, due to destructive effect of machining, can deviate heavily from the magnetic material producer's data. This side effect can usually be mitigated by additional annealing of the magnetic circuit elements according to the procedure recommended by the producer, however, the finally generated magnetic field by the whole circuit requires experimental verification. Typically, to verify the magnetic circuit only the field magnitude is compared with the simulations [9], [10], because determination of the highly curved field lines found in the Hall thrusters is more complex and time-consuming. The topography can be rendered rather easily with the use of iron fillings as in [11], however, this method makes it hard to compare with simulations. In [12] the authors perform a 2D mapping of the magnetic field using a 3-axis gaussmeter and automated test-bench, although the field topography is presented only in the form of a vector map which additionally does not seem to match the simulated field lines (possibly a plotting error). On the other hand, in [13] the measured field lines are shown, but only in a limited region of small field curvature where the streamlines approach (integrating along a chosen line) can be safely used.

In this work the magnetic circuit designed in IFPiLM (Instytut Fizyki Plazmy i Laserowej Mikrosyntezy - Institute of Plasma Physics and Laser Microfusion) is measured, the field topography is determined and the results are compared with simulations. A robust analytical procedure for experimental determination of magnetic field topography in Hall thrusters is presented. The method, based on the Stokes stream function approach, well suited for cylindrically symmetric geometries like that of a Hall thruster, works for even highly cusped topographies and provides quantitatively meaningful field lines (i.e., with known magnetic flux step) as opposed to the streamlines approach.

\section{EXPERIMENTAL SETUP}

\section{A. MAGNETIC CIRCUIT}

The magnetic circuit characterized in this work was prepared for a small (discharge channel outer diameter of $50 \mathrm{~mm}$, channel width of $8 \mathrm{~mm}$ ) Hall thruster developed in IFPiLM in the scope of the project HIKHET (High Impulse Krypton Hall Effect Thruster). Most of the circuit was made from pure iron (ALLIEDPUREIRON) with the exception of inner and outer magnetic poles, which were made from ironcobalt alloy (Hiperco 50A / Iron-Cobalt ASTM-A801-09) to be able to withstand higher temperatures observed there during operation of the thruster (Curie temperature of ironcobalt alloys exceeds that of pure iron by around $170^{\circ} \mathrm{C}$ while saturation of the magnetization curve by about $0.2 \mathrm{~T}$ at room temperature). All elements were annealed to restore their magnetic properties after manufacturing. Parts from pure iron were annealed in hydrogen, and iron-cobalt elements in high vacuum (approximately $1 \cdot 10^{-5} \mathrm{mbar}$ ). The temperature profiles of the processes were set according to the recommendations of the material manufacturers. To limit corrosion, pure iron elements were additionally nickel electroplated $(0.3 \mu \mathrm{m})$. Magnetic field was generated by five coils (one inner and four outer), each wound with 92 turns of $\varnothing 1 \mathrm{~mm}$ copper wire, which were driven by Hua Yi Electronics HY3005D-2 power supply.

The circuit was designed to allow testing of two qualitatively different topographies of magnetic field, named here as classical (provided by magnetic circuits of wellknown Hall thrusters of SPT-100 class) and zero-B (characterized by a reversal of the field direction inside the channel, manifested by a point of zero magnetic field). As is schematically shown in Fig.2., changing between the two configurations was done with an iron insert by opening (removing the insert, thus introducing a slit) or shorting (putting the insert back into the slit) the magnetic circuit at the back of the thruster. Physical meaning of both topographies from the point of view of thruster operation is 
out of the scope of this paper and will not be discussed here (however, interested reader is kindly referred to the following papers [14]-[17]). The expected magnetic field was modelled for different coil currents using commercial Finite Element Method software ANSYS Maxwell 16.0. Due to the lack of full axial symmetry (four outer coils) the magnetic circuit was simulated in $3 \mathrm{D}$, incorporating periodic boundary conditions (for 2D problems a non-commercial FEMM is a popular, freeto-use alternative). Computational mesh was generated and refined automatically by ANSYS Maxwell, with the additional constraint of $2 \mathrm{~mm}$ on the maximum length of elements. Because the magnetization curves of the materials were not measured after the annealing, for the purpose of the simulation the B-H curves for Hiperco 50A and Electric Iron from Carpenter company were used. This was expected to be one of the sources of error when comparing with the measurements.

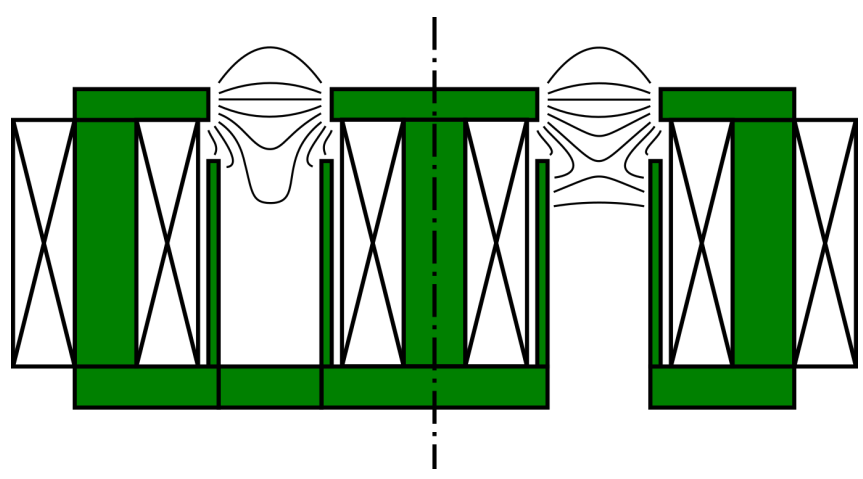

Fig.2. Two distinct topographies possible to achieve in the tested magnetic circuit: classical on the left and zero-B on the right (the change is made by an iron insert at the rear). Ferromagnetic elements of the magnetic circuit are drawn in green and excitation coils are shown as crossed rectangles.

\section{B. GAUSSMETER}

All measurements of the magnetic field were performed using commercial RX-25 Hall effect gaussmeter by Resonance Technology. Probe of the gaussmeter was a rod $100 \mathrm{~mm}$ long and $\varnothing 4 \mathrm{~mm}$ thick containing three miniature Hall effect GaAs type sensors measuring magnetic field induction along orthogonal axes with declared accuracy of $\pm(0.5 \% \cdot \mathrm{rdg}+2 \mathrm{dgt})$. Because the sensors were necessarily translated relative to each other inside the probe and also because there were concerns if the system was truly orthogonal, the gaussmeter was additionally recalibrated in three steps to improve the accuracy of the measurements.

First, the exact position of the sensors (assuming $Y$ direction along the probe - Fig.3.) $y_{X}, y_{Y}, y_{Z}$ was determined with a magnetized sharpened iron rod, by moving it along the gaussmeter probe with a step of $0.1 \mathrm{~mm}$ and reading values displayed by the sensors. Translation with respect to the tip of the probe $y_{\text {probe }}$ of individual sensors $\left(y_{X 0}, y_{Y 0}, y_{Z 0}\right)$ was the position corresponding to the maximum or zero displayed value (depending on the alignment of the sensor). Thus, the measured translation was $y_{X 0}=3.8 \mathrm{~mm}, y_{Y 0}=2.1 \mathrm{~mm}, y_{Z 0}$ $=5.4 \mathrm{~mm}$.

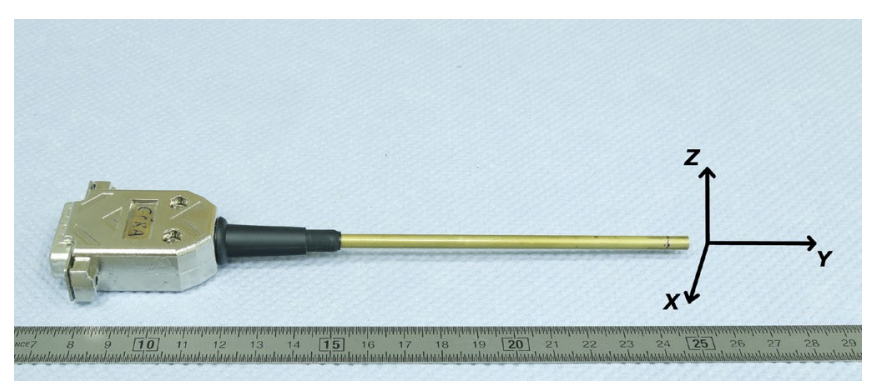

Fig.3. Probe of the gaussmeter. Assumed coordinate system and a ruler are shown for reference.

Then, the rotation of each sensor was determined inside a Helmholtz coil (manufactured in IFPiLM - Fig.4.) by searching for an angular position which corresponded to zero displayed value, i.e. magnetic field lying in the plane perpendicular to the sensor direction. The angle was measured with a protractor with $1^{\circ}$ resolution. This was done in two planes for each sensor to obtain the actual rotation in 3D. These projections were next rewritten to a normalized, non-orthogonal basis $\mathbb{A}$ in which each basis vector $\hat{x}, \hat{y}, \hat{z}$ represented the spatial direction of one sensor:

$$
\mathbb{A}=\left[\begin{array}{c}
\hat{x} \\
\hat{y} \\
\hat{z}
\end{array}\right]=\left[\begin{array}{c}
\frac{\left(1, \tan x_{X Y}, \tan x_{X Y} \tan x_{Y Z}\right)}{\left\|\left(1, \tan x_{X Y}, \tan x_{X Y} \tan x_{Y Z}\right)\right\|} \\
\frac{\left(1, \tan y_{X Y}, \tan y_{X Y} \tan y_{Y Z}\right)}{\left\|\left(1, \tan y_{X Y}, \tan y_{X Y} \tan y_{Y Z}\right)\right\|} \\
\frac{\left(1, \tan z_{X Y}, \tan z_{X Y} \tan z_{Y Z}\right)}{\left\|\left(1, \tan z_{X Y}, \tan z_{X Y} \tan z_{Y Z}\right)\right\|}
\end{array}\right],
$$

where $x_{X Y}=2^{\circ}, x_{Y Z}=93^{\circ}, y_{X Y}=90^{\circ}, y_{Y Z}=1^{\circ}, z_{X Y}=9^{\circ}$, and $Z_{Y Z}=89^{\circ}$ (here indices $X Y$ and $Y Z$ denote the plane in which the rotation was measured, with $0^{\circ}$ meaning, respectively, $X$ or $Y$ direction, and $90^{\circ} Y$ or $Z$ direction). It can be seen that the $Y$ sensor, which was parallel to the probe, had been the best aligned of the three.

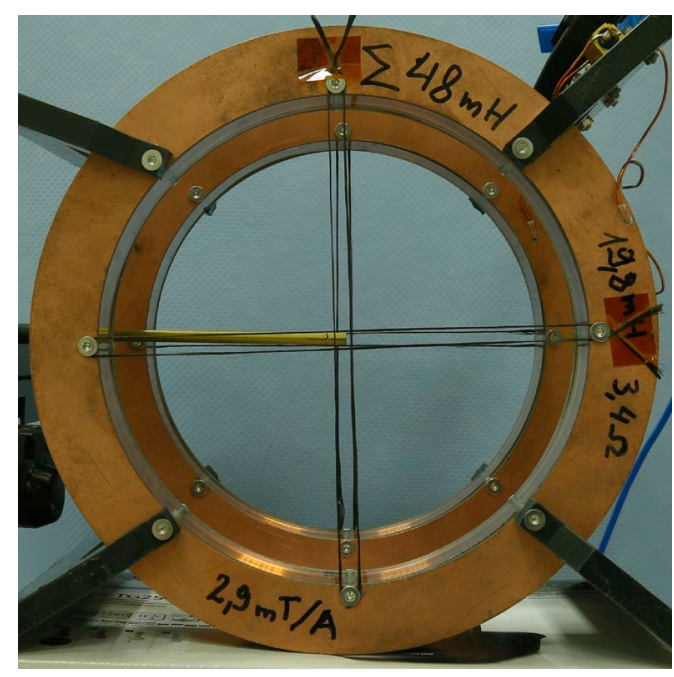

Fig.4. Gaussmeter readied inside the Helmholtz coil. Zero displayed value was searched by rotating the coil. 
Finally, in order to restore the factory calibration of the gaussmeter which had been made with the assumption that all the Hall effect sensors were orthogonal, with the use of the same Helmholtz coil an additional calibration factor was introduced. This factor was determined individually for each sensor as the ratio of magnetic field magnitude inside the Helmholtz coil before and after correcting for rotation: $k_{X}=$ $0.834, k_{Y}=1.000, k_{Z}=1.027$ (because sensor $Y$ was not rotated much, it needed virtually no magnitude correction). Values similar to $6 \%$ were obtained also from calibration using IFPiLM's own Helmholtz coil. This difference, together with declared accuracy of the instrument, was used to estimate the final uncertainty of the results.

To summarize, having the exact positions and orientations of the three sensors $X, Y, Z$, all subsequent readings were corrected in the following way:

1) correction for translation: $\left[\begin{array}{l}y_{X} \\ y_{Y} \\ y_{Z}\end{array}\right]=y_{\text {probe }}-\left[\begin{array}{l}y_{X 0} \\ y_{Y 0} \\ y_{Z 0}\end{array}\right]$;

2) correction for rotation: $\mathbf{B}_{\text {rot_corr }}=\mathbb{A}^{-1} \mathbf{B}_{\text {probe }}$;

3) correction for magnitude: $\mathbf{B}_{c o r r}=\left[\begin{array}{ccc}k_{X} & 0 & 0 \\ 0 & k_{Y} & 0 \\ 0 & 0 & k_{Z}\end{array}\right] \mathbf{B}_{\text {rot_corr }}$.

\section{METHODOLOGY}

The magnetic circuit was assembled in the desired configuration (with or without a slit) and the bare thruster (without channel insulator, cathode, etc.) was fixed to a desk with a vice. A laser level was used to assure parallelism of thruster axis and desk surface. Next, the probe of the gaussmeter was fixed to a manual precision positioning table and was aligned along the thruster axis in such a way as to move only in the $(\rho, z)$ plane of the thruster, as shown in Fig.5. The misalignment of the thruster and the probe was estimated to be no larger than $2^{\circ}$ in each direction. Prior to the measurements the probe was zeroed in a dedicated zero field chamber provided by the manufacturer.

The magnetic field was measured in four configurations, presented in Table 1. The coil currents ratio chosen (1.6) was one that resulted in the most symmetric magnetic field topography in both circuit configurations, according to ANSYS Maxwell calculations (symmetry of the field was assessed using integral criterion along the channel centreline $\left.\int\left|\frac{B_{Z}}{B_{z, \max }}\right| d z=\min \right)$. For each configuration over 2000 points were acquired by mapping the channel interior and exterior in a grid with step of $0.5 \mathrm{~mm}$.

The gaussmeter readings were corrected for translation and rotation of the three separate Hall effect sensors with the use of a Helmholtz coil (according to the calibration procedure described above). Because the positions of the sensors relative to each other were not multiples of grid step, the data was then interpolated and smoothed with local polynomial surface fitting (LOESS) to obtain all three components of the magnetic field in each $(\rho, z)$ point. In case of the regions where the measurements from all three sensors did not overlap, the data was discarded.

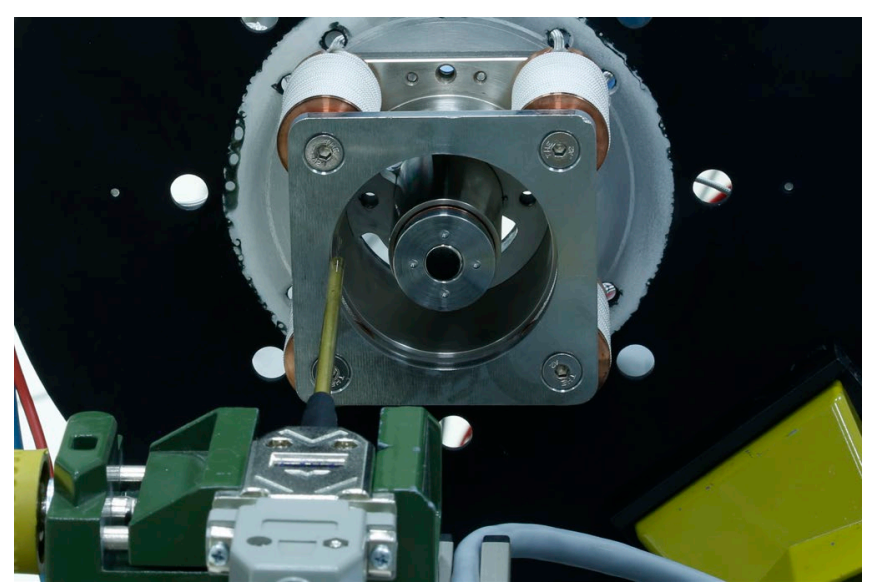

Fig.5. Gaussmeter mounted on a positioning table and ready for measurement of classical topography (iron insert is visible at the channel bottom).

Table 1. Studied configurations of magnetic field.

\begin{tabular}{|c|c|c|}
\hline case & magnetic circuit & $\begin{array}{c}\text { coil currents } \\
\text { (inner : outer) }\end{array}$ \\
\hline 1 & with insert & $4.0 \mathrm{~A}: 2.5 \mathrm{~A}$ \\
\hline 2 & (classical topography) & $8.0 \mathrm{~A}: 5.0 \mathrm{~A}$ \\
\hline 3 & with slit & $4.0 \mathrm{~A}: 2.5 \mathrm{~A}$ \\
\cline { 3 - 3 } & (zero B topography) & $8.0 \mathrm{~A}: 5.0 \mathrm{~A}$ \\
\hline
\end{tabular}

Having the corrected and interpolated values of the three components of magnetic field $-B_{\rho}, B_{\varphi}, B_{z}$, the results could already be compared with the outcomes of simulations along several chosen lines (e.g., along channel centreline). However, to compare the whole topography, it was necessary to retrieve magnetic field lines. The most obvious method for this was the streamline approach, in which experimental data is integrated (for example, using the 4th-order Runge-Kutta method) from wall to wall along several chosen field lines. However, while yielding satisfactory results for simple topographies, for highly cusped fields this integration procedure is cumbersome and prone to large errors when one of the components changes sign (as was the case in zero-B topography). That is why a more robust Stokes stream function approach was chosen.

In this approach it can be shown that, under the assumption of divergence-free vector field and cylindrical symmetry, the continuity equation is satisfied by a scalar function $\psi(\rho, z)$ called stream function, which is constant along a streamline [18]. In case of magnetic field the first assumption is satisfied automatically by the Gauss's law $\nabla \cdot \mathbf{B}=0$. The second assumption is also satisfied with good precision in the region of interest by the fact that Hall thrusters are deliberately designed to have as cylindrically symmetric field as possible (in order to mitigate plasma instabilities which may arise on non-uniformities). Following this approach, the stream function for magnetic field can be expressed as $\psi=\rho A_{\varphi}$, where $A_{\varphi}$ is the azimuthal component of magnetic vector potential. On the other hand, the definition $\mathbf{B}=\nabla \times \mathbf{A}$ results in $B_{Z}=\frac{1}{\rho} \frac{\partial\left(\rho A_{\varphi}\right)}{\partial \rho}$ and $B_{\rho}=-\frac{\partial A_{\varphi}}{\partial z}$, which then become, using 
the stream function, $B_{z}=\frac{1}{\rho} \frac{\partial \psi}{\partial \rho}$ and $B_{\rho}=-\frac{1}{\rho} \frac{\partial \psi}{\partial z}$, respectively. Consequently, $\psi$ can be obtained in the whole $(\rho, z)$ space by integrating the measurements in two directions: $\psi=$ $\int-\rho B_{\rho} d z$ and $\psi=\int \rho B_{z} d \rho$.

In practice, the integration was done using the 4th-order Runge-Kutta method, taking the initial value of $\psi_{0}=0$. Because of the errors naturally cumulating during the integration (resulting from measurement uncertainty and finite spatial resolution), in order to obtain the best accuracy, the point of $\psi_{0}$ should be chosen near the region of primary interest - in our case it was the intersection of channel centreline and exit plane. The order of integration was arbitrarily chosen to be first along the $z$ direction and then along $\rho$. Finally, having the values of $\psi$ in the interesting region and knowing that they should be constant along the field lines, the magnetic field topography was drawn simply as a contour plot of $\psi$.

\section{RESULTS AND DISCUSSION}

In Fig.6. and Fig.7. presented are the profiles of three components of the magnetic field (radial $B_{\rho}$, azimuthal $B_{\varphi}$, and axial $B_{z}$ ) along the channel centreline (at radius of $21 \mathrm{~mm}$ ) and along the poles centreline (at axial position of $-2.4 \mathrm{~mm}$, assuming $0 \mathrm{~mm}$ is the thruster exit plane). Raw, uncorrected data from the gaussmeter is shown as a reference besides the corrected values and the outcomes from the simulation. Expanded uncertainty $(\mathrm{k}=2)$ is shown as shaded area around the corrected data.

The best agreement between measurements and simulations was found for the radial component of the magnetic field. Profiles along channel and poles centrelines were well preserved for both classical and zero-B topographies, and in the most important region (from $Z=$ $-10 \mathrm{~mm}$ to $z=0 \mathrm{~mm}$ ) the magnitude differed by less than the gaussmeter accuracy. On the other hand, deep inside the channel in the classical case the gaussmeter readings crossed zero which should not occur for this topography - this was probably due to incorrect zeroing of the probe. The azimuthal component at a first glance was very different from the nearzero values predicted in simulations. However, the experimental profiles of azimuthal component showed strong resemblance to the respective profiles of radial component (along channel centreline as well as along poles centreline) with the exception that in the case of classical topography the correlation was negative and in the case of zero-B the correlation was positive. This can be explained by an up or down shift or rotation of the $(\rho, z)$ measurement plane with respect to the thruster middle plane resulting in the azimuthal sensor measuring some of the radial value.

For example, the value of azimuthal component at maximum for classical 8.0:5.0 case was approximately $2.5 \%$ of the radial component value, which would require a rotation of the measurement plane by only $1.5^{\circ}$. This is within the accuracy with which the probe could be manually positioned in our setup.

The experimental shapes of the axial component correlated quite well with the simulations as well, although the discrepancy of the magnitude relatively grew downstream of the thruster (positive $z$ direction). Similarly to the azimuthal component, the reason for this could be the tilt of the $(\rho, z)$ measurement plane. Also, it is worth noting that some effects of the machining (threads, chamfers, slits, and cavities) were not included in the simulation model, which might have been an additional source of discrepancy for all three components. However, briefly testing the dependence of simulation outcomes on the inclusion of such imperfections, it became evident that the impact is not significant.
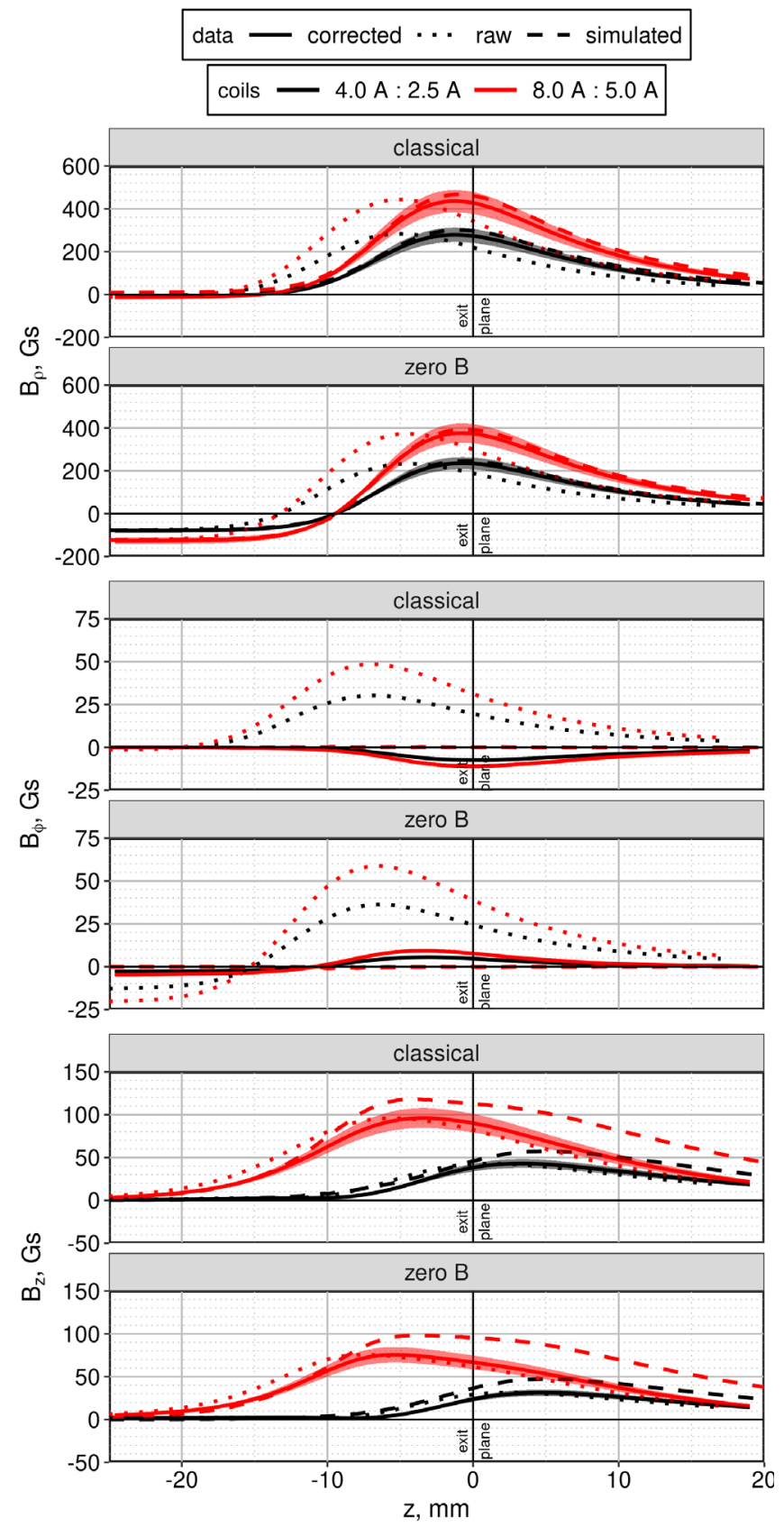

Fig.6. Radial, azimuthal and axial component of the magnetic field along the channel centreline $(\rho=21 \mathrm{~mm})$. Dashed lines were simulated with ANSYS Maxwell. Dotted and solid lines were measured with gaussmeter. Solid lines are accompanied by shaded area representing expanded uncertainty $(\mathrm{k}=2)$. 

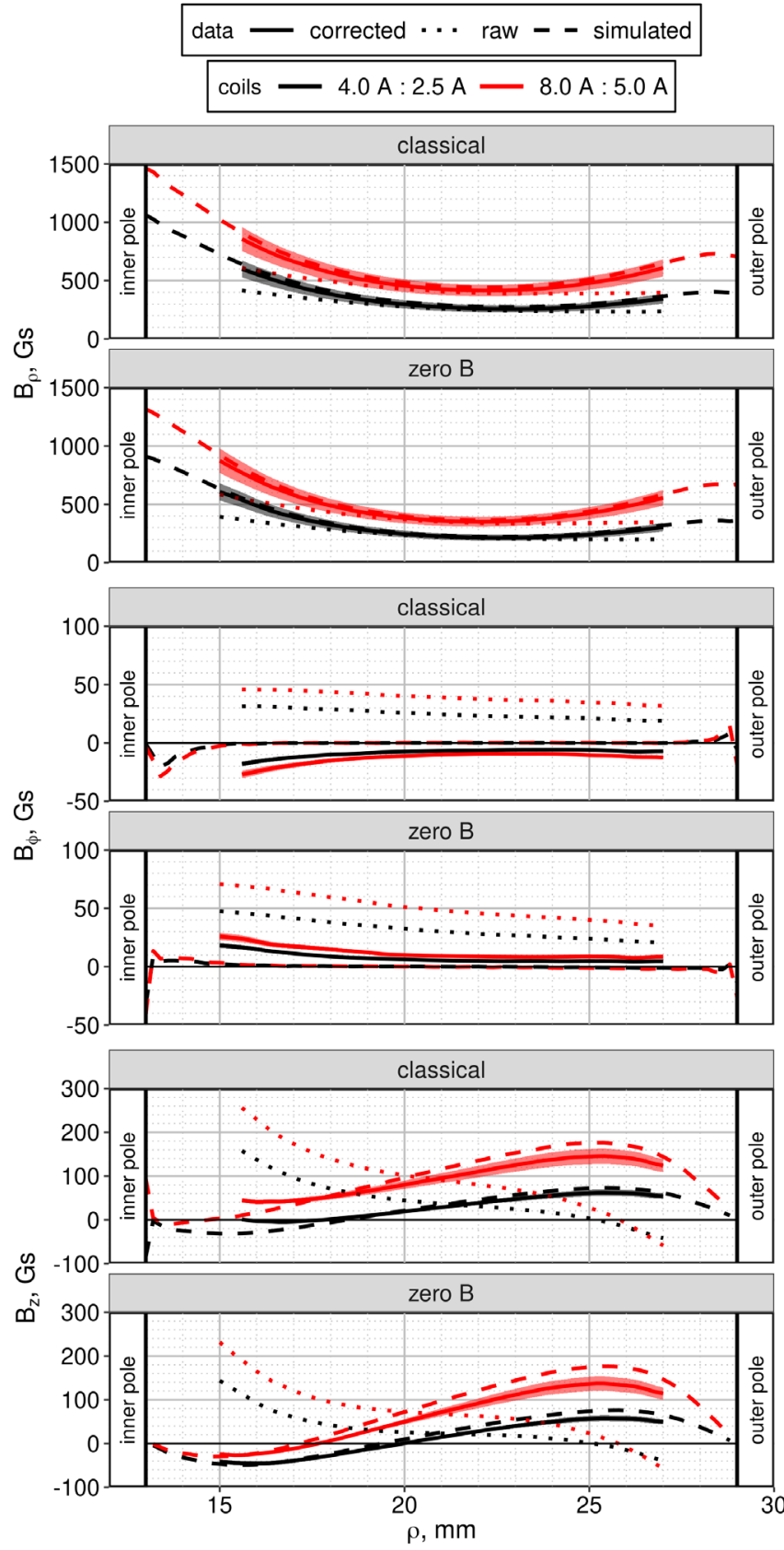

Fig.7. Radial, azimuthal and axial component of the magnetic field along the poles centreline $(z=-2.4 \mathrm{~mm})$. Dashed lines were simulated with ANSYS Maxwell. Dotted and solid lines were measured with gaussmeter. Solid lines are accompanied by shaded area representing expanded uncertainty $(\mathrm{k}=2)$.

The field topographies, retrieved according to the stream function approach described earlier, are shown in Fig.8. for simulations (colour maps of field magnitude and dashed field lines) and gaussmeter measurements (solid field lines). The stream function is plotted as contour lines with a constant interval of $10^{4} \mathrm{Gs} \mathrm{mm}^{2}$, and in this way can be interpreted as the usual magnetic field lines (i.e., higher line density corresponds to a larger magnitude of the field).

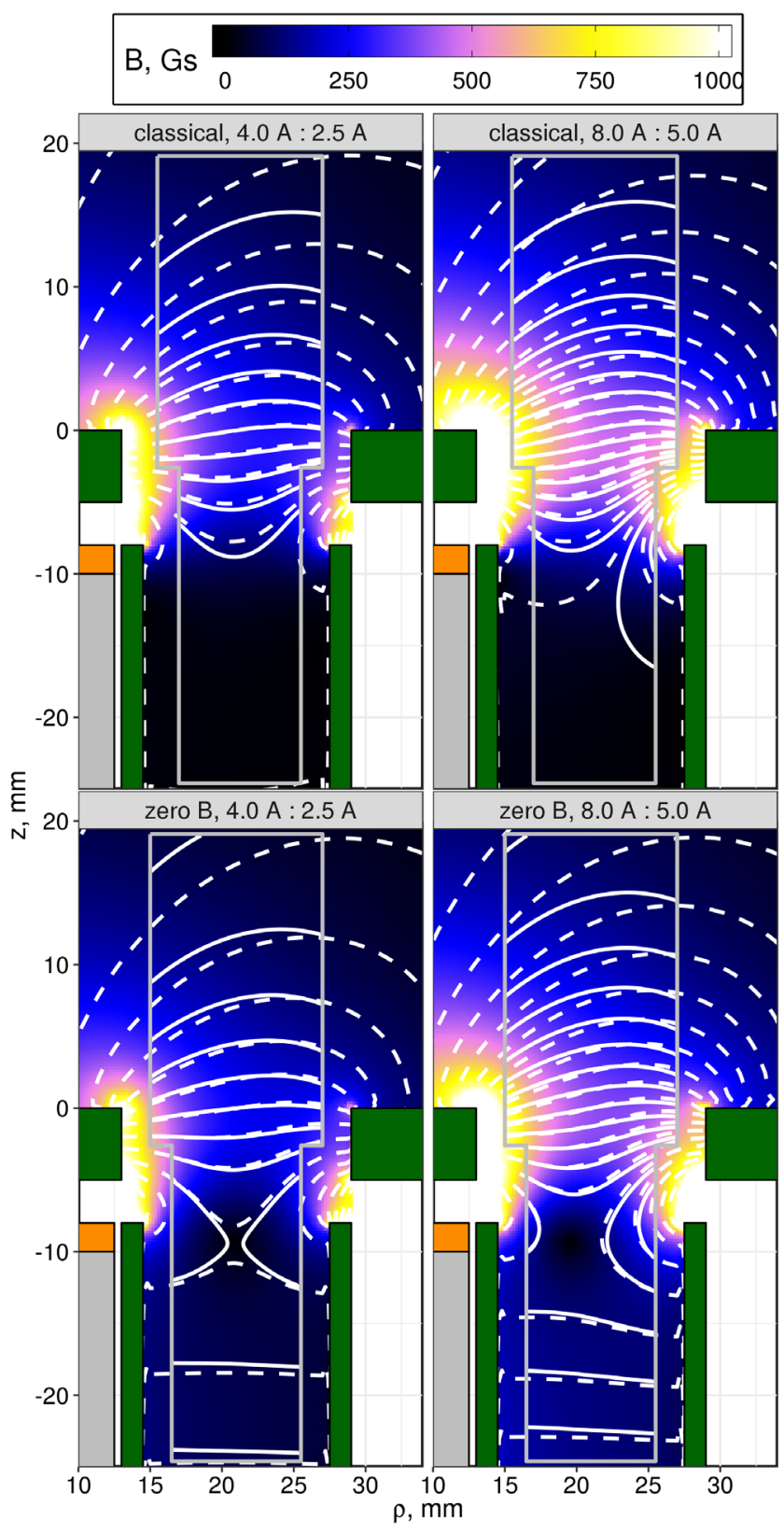

Fig.8. Magnetic field topography inside the thruster (with a slit or an insert) for different coil-current ratios. Colour maps of magnetic field magnitude and dashed field lines were simulated with ANSYS Maxwell. Solid magnetic field lines were measured with gaussmeter.

The obtained magnetic field topographies showed very good agreement with one another. Only in the region downstream of the exit plane the field lines inclination started to deviate slightly, which was a consequence of discrepancy in values of axial component between simulations and measurements. However, it should be pointed out that the magnetic field that far outside of the channel is less significant from the point of view of thruster operation. On the other hand, the most important features were well reproduced in all four cases. For example, the location of a 
region of zero-B inside the channel was accurately determined. Also, the shift of the field towards the inner wall for larger coil currents and consequent tilting of the field lines (related to the idea of the so-called magnetic lens) was consistent with the simulations. It is worth noting that for high values of the magnetization current $(8.0 \mathrm{~A}: 5.0 \mathrm{~A})$ the magnetic circuit operates in the nonlinear range of the magnetization curve that is reflected by significantly different relevant topographies of B-field when compared to the cases with lower magnetization currents.

As can be seen, due to the dimensions of the probe, the magnetic field near the poles and screens of the magnetic circuit could not be measured. However, it should be noted that for normal operation the thruster is fitted with a channel insulator (diamagnetic boron nitride ceramics) which narrows the channel that confines the plasma to $17 \mathrm{~mm}$ and $25 \mathrm{~mm}$ (inner and outer wall radius, respectively). Consequently, in practice the knowledge of the topography right next to the elements of the magnetic circuit is unimportant.

\section{CONCLUSIONS}

The verification of magnetic field topography is important in the light of recent efforts at optimization of the magnetic field and designing of increasingly more complex circuits. Such measurements can minimize the danger of misinterpreting the experimental outcomes as well as improve predictive accuracy of numerical models simulating the discharge in the Hall thruster.

In this paper it has been shown how the Stokes stream function approach may be used to reliably determine magnetic field topography in a small Hall thruster, an electric propulsive engine for spacecraft. The method is well suited to these devices thanks to the inherent symmetry of their design and divergence-free of the magnetic field itself, which satisfy the necessary assumptions of this approach. This robust technique allows to accurately plot the magnetic field lines even for a highly cusped topography, without the need of advanced integration schemes like in the streamlines approach, making it ideal to study even the most complex designs of magnetic circuit.

To reliably reproduce the topography, the measurements need to be taken with good spatial resolution (here, $0.5 \mathrm{~mm}$ was sufficient) which is necessarily time-consuming. Although in this work a manual positioning table was sufficient to obtain accurate data, the measurement process could be considerably sped up with the use of an automated XY stage. Moreover, the gaussmeter needs to be precisely calibrated and the measurement plane of the probe accurately positioned with respect to the thruster in order to obtain reliable data.

Because most often each Hall thruster is a different device with a unique magnetic circuit, the results obtained for IFPiLM's model cannot be directly compared with magnetic field topographies of other thrusters. However, the outcomes obtained with the above method contain more information about the topography than vector maps presented in [12] (which do not discriminate between field lines) or streamlines presented in [13] (which can be easily drawn only in simple topographies). The field lines obtained with the Stokes stream function approach are spaced with known magnetic flux step, are accurately reconstructed even for highly cusped topographies and are identifiable by a single scalar quantity (i.e., each field line has a unique numerical value). This last property can be useful in various numerical tasks, like analysis of guiding centre movement along the field line or magnetic mirror calculations where for each point in the channel a corresponding point at the wall lying on the same field line must be found.

\section{ACKNOWLEDGMENT}

The work presented in this article was carried out in the scope of the project "High Impulse Krypton Hall Effect Thruster" funded by the European Space Agency under the programme Polish Industrial Incentive Scheme (grant agreement no. 4000122415/17/NL/GE). The view expressed by the authors can in no way be taken to reflect the official opinion of the European Space Agency.

\section{REFERENCES}

[1] Mazouffre, S. (2016). Electric propulsion for satellites and spacecraft: Established technologies and novel approaches. Plasma Sources Science and Technology, 25, 033002. doi: 10.1088/0963-0252/25/3/033002.

[2] Levchenko, I., Bazaka, K., Ding, Y., Raitses, Y., Mazouffre, S., Henning, T., Klar, P.J., Shinohara, S., Schein, J., Garrigues, L., Kim, M., Lev, D., Taccogna, F., Boswell, R.W., Charles, C., Koizumi, H., Shen, Y., Scharlemann, C., Keidar, M., Xu, S. (2018). Space micropropulsion systems for Cubesats and small satellites: From proximate targets to furthermost frontiers. Applied Physics Reviews, 5, 011104. doi: 10.1063/1.5007734.

[3] Levchenko, I., Keidar, M., Cantrell, J., Wu, Y.-L., Kuninaka, H., Bazaka, K., Xu, S. (2018). Explore space using swarms of tiny satellites. Nature, 562, 185-187. doi: 10.1038/d41586-018-06957-2

[4] Levchenko, I., Xu, S., Wu, Y.-L., Bazaka, K. (2020). Hopes and concerns for astronomy of satellite constellations. Nature Astronomy, 4, 1012-1014. doi: 10.1038/s41550-020-1141-0.

[5] Goebel, D.M., Katz, I. (2008). Fundamentals of Electric Propulsion. John Wiley \& Sons.

[6] Morozov, A.I. (2003). The conceptual development of stationary plasma thrusters. Plasma Physics Reports, 29, 235-250. doi: 10.1134/1.1561119.

[7] Boeuf, J.P. (2017). Tutorial: Physics and modeling of Hall thrusters. Journal of Applied Physics, 121, 011101. doi: 10.1063/1.4972269.

[8] Levchenko, I., Xu, S., Mazouffre, S., Lev, D., Pedrini, D., Goebel, D., Garrigues, L., Taccogna, F., Bazaka, K. (2020). Perspectives, frontiers, and new horizons for plasma-based space electric propulsion. Physics of Plasmas, 27, 020601. doi: 10.1063/1.5109141.

[9] Hofer, R.R., Haas, J.M., Peterson, P.Y., Martinez, R.A., Gallimore, A.D. (2000). Optimization of Hall thruster magnetic field topography. In 27th IEEE International Conference on Plasma Science. IEEE, doi: 10.1109/PLASMA.2000.855077. 
[10] Manzella, D., Jankovsky, R., Hofer, R.R. (2002). Laboratory model $50 \mathrm{~kW}$ Hall thruster. In 38th AIAA/ASME/SAE/ASEE Joint Propulsion Conference \& Exhibit. AIAA, doi: 10.2514/6.2002-3676.

[11] Mitrofanova, O.A., Gnizdor, R.Y. (2013). Influence of SPT magnetic field on life time characteristics of the thruster. In 33rd International Electric Propulsion Conference (October 7-10, 2013, Washington, DC, USA).

[12] Garrigues, L., Mazouffre, S., Henaux, C., Vilamot, R., Rossi, A., Harribey, D., Bourgeois, G., Vaudolon, J., Zurbach, S. (2013). Design and first test campaign results with a new flexible magnetic circuit for a Hall thruster. In 33rd International Electric Propulsion Conference (October 7-10, 2013, Washington, DC, USA).

[13] Mikellides, I.G., Katz, I., Hofer, R.R., Goebel, D.M. (2014). Magnetic shielding of a laboratory Hall thruster. I. Theory and validation. Journal of Applied Physics, 115, 043303. doi: 10.1063/1.4862313.

[14] Kim, V.P., Gnizdor, R.Y., Grdlichko, D.P., Merkuriev, D.V., Mitrofanova, O.A., Smirnov, P.G., Shilov, E.A., Zakharchenko, V.S. (2018). Fundamental principles employed for ionization and acceleration layer control in the discharge of a stationary plasma thruster. Journal of Surface Investigation, 12, 1237-1247. doi: 10.1134/S1027451018050610.
[15] Hofer, R.R., Peterson, P.Y., Gallimore, A.D. Jankovsky, R.S. (2001). A High specific impulse twostage Hall thruster with plasma lens focusing. In 27th International Electric Propulsion Conference (October 15-19, 2001, Pasadena, CA, USA).

[16] Grimaud, L., Mazouffre S. (2018). Performance comparison between standard and magnetically shielded $200 \mathrm{~W}$ Hall thrusters with $\mathrm{BN}-\mathrm{SiO} 2$ and graphite channel walls. Vacuum, 155, 514-523. doi: 10.1016/j.vacuum.2018.06.056.

[17] Ding, Y., Li, P., Zhang, X., Wei, L., Sun, H., Peng, W., Yu, D. (2017). Effects of the magnetic field gradient on the wall power deposition of Hall thrusters. Journal of Plasma Physics, 83, 905830205. doi: 10.1017/S0022377817000241.

[18] Batchelor, G.K. (1967). An Introduction to Fluid Dynamics. Cambridge University Press, p. 75-79.
Received August 19, 2021 Accepted September 10, 2021 